\title{
Improved Lower Bounds on the Size of Balls over Permutations with the Infinity Metric
}

\author{
Moshe Schwartz, Senior Member, IEEE, and Pascal O. Vontobel, Senior Member, IEEE
}

\begin{abstract}
We study the size (or volume) of balls in the metric space of permutations, $S_{n}$, under the infinity metric. We focus on the regime of balls with radius $r=\rho \cdot(n-1), \rho \in[0,1]$, i.e., a radius that is a constant fraction of the maximum possible distance. We provide new lower bounds on the size of such balls. These new lower bounds reduce the asymptotic gap to the known upper bounds to at most 0.029 bits per symbol. Additionally, they imply an improved ball-packing bound for error-correcting codes, and an improved upper bound on the size of optimal covering codes.
\end{abstract}

Index Terms-Asymptotic gap, infinity metric, permanent, permutation, rank modulation, Sinkhorn theorem.

\section{INTRODUCTION}

$\mathbf{G}$ IVEN a metric space $(M, d)$, perhaps one of the most basic constructs is that of a ball

$$
B_{r}(x) \triangleq\left\{x^{\prime} \in M \mid d\left(x, x^{\prime}\right) \leqslant r\right\},
$$

where $x \in M$ is the ball's center and $r$ is the ball's radius. Since many coding-theoretic problems may be viewed as the study of packing or covering of a metric space by balls, properties of balls and their parameters have been studied extensively in a wide range of metrics [10], [11], [33].

An important feature of a ball is its size (or volume), i.e., the number of points in the ball. It is an important component in many bounds on code parameters, most notably, the ballpacking bound and the Gilbert-Varshamov bound [33]. Thus, the exact size, the asymptotic size, or bounds on the size of balls in various metrics are of interest.

Lately, metric spaces over permutations have received increased attention. This is motivated, in particular, by the recent application of rank modulation to non-volatile memory systems [24]: in such applications, the charge levels of memory cells are compared against each other, and a permutation is induced by the relative ranking of the cells' charge levels. For designing error-correcting codes or covering codes over the space of permutations, one needs to choose a suitable metric and so several metrics have been studied for the space of

The material in this paper was presented in part at the IEEE International Symposium on Information Theory (ISIT 2015), Hong Kong, China SAR, June 2015 [38].

Moshe Schwartz is with the Department of Electrical and Computer Engineering, Ben-Gurion University of the Negev, Beer Sheva 8410501, Israel (e-mail: schwartz@ee.bgu.ac.il).

Pascal O. Vontobel is with the Department of Information Engineering, The Chinese University of Hong Kong, Shatin, N.T., Hong Kong, (e-mail: pascal.vontobel@ieee.org).

This work was supported in part by the Israel Science Foundation (ISF) grant No. 130/14.

Submitted to IEEE Transactions on Information Theory, Sep. 16, 2016. Revised on Feb. 8, 2017. Accepted on Apr. 2, 2017. permutations, including Hamming's metric [1], [3], [4], [7], [8], [13], [14], [26], [35], Kendall's $\tau$-metric [2], [5], [6], [9], [25], [34], [46], [49], and Ulam's metric [18], [19].

This paper focuses on the infinity metric (whose formal definition will follow in the next section), which is motivated by applications to rank modulation in some non-volatile memory systems (e.g., flash memory or phase-change memory systems). Recall that in a flash memory system, each cell has a charge level which can be changed or read out by a suitable circuit. The idea behind rank modulation is that information is encoded in terms of the rank of the charge values. More precisely, consider $n$ flash cells, each with some charge level. Let $f_{1}$ be the rank of the charge level of the first cell, $f_{2}$ be the rank of the charge level of the second flash cell, etc. The information encoded in these $n$ flash cells corresponds to the permutation $\left(f_{1}, f_{2}, \ldots, f_{n}\right)$. However, charge levels may be set imprecisely during the storing process, they may change over time due to physical processes, or they may be read out imprecisely due to noise, thereby giving rise to a distorted permutation $\left(f_{1}^{\prime}, f_{2}^{\prime}, \ldots, f_{n}^{\prime}\right)$. In a typical setting, we may have some bound on the charge-level distortion, which translates to a bound on $d \triangleq \max _{i}\left|f_{i}-f_{i}^{\prime}\right|$, i.e., each cell may change its rank by a limited amount. We say $d$ is the distance between the two permutations under the infinity metric. With a metric space in hand we can combat such distortion by designing suitable error-correcting codes.

More generally, spaces of permutations with the infinity metric have been used for error-correction [30], [39], [42], [49], code relabeling [43], anticodes [37], covering codes [17], [46], and snake-in-the-box codes [47], [48]. It is therefore surprising that the asymptotic size of a ball in this metric space is (to the best of our knowledge) unknown, and a considerable gap exists between the known lower and upper bounds.

The goal of this paper is to reduce the gap between the lower and upper bounds on the asymptotic size of balls in the space of permutations with the infinity metric. To that end, we exploit a well-known connection between the size of the aforementioned balls, and permanents of binary Toeplitz matrices. We carefully employ lower bounds on permanents of non-negative matrices to obtain the desired results. (One of these bounds is well known, one is somewhat recent.)

The paper is organized as follows. In Section $\Pi$ we present notations and definitions. Result-wise, the main section is Section III. where we collect not only known, but also our new results on the asymptotic gap between upper and lower bounds. Whereas in Section IV we discuss how the known results in Section $\Pi 1 \mathrm{II}$ are obtained, we devote Section $\mathrm{V}$ to the presentation of the new lower bounds that lead to the new 
results in Section III. We conclude the paper in Section VI

\section{NOTATION}

For the rest of this paper, $n$ will denote a positive integer. With this, we define $[n] \triangleq\{1,2, \ldots, n\}$ and let $S_{n}$ be the set of all permutations over $[n]$. The identity permutation in $S_{n}$ is denoted by $\operatorname{Id}_{n}$. Additionally, the composition of any two permutations $f, g \in S_{n}$ is denoted by $f g$ and represents the mapping $i \mapsto f(g(i))$.

For any $f, g \in S_{n}$, the infinity metric (or infinity distance) between them, denoted $d_{\infty}(f, g)$, is defined as

$$
d_{\infty}(f, g) \triangleq \max _{i \in[n]}|f(i)-g(i)| .
$$

Since $d_{\infty}(\cdot, \cdot)$ is the only metric we will be using, we shall simply denote it by $d(\cdot, \cdot)$. Observe that for any $f, g \in S_{n}$, we have $0 \leqslant d(f, g) \leqslant n-1$.

We define the ball of radius $r$ centered at $f \in S_{n}$ as the set

$$
B_{r, n}(f) \triangleq\left\{g \in S_{n} \mid d(f, g) \leqslant r\right\} .
$$

The infinity metric over $S_{n}$ is right invariant [12], i.e., for all $f, g, h \in S_{n}$ we have $d(f h, g h)=d(f, g)$. Thus, the size of a ball depends only on $r$ and $n$, and not on the choice of the center. We will therefore denote by $\left|B_{r, n}\right|$ the size of a ball of radius $r$ in $S_{n}$.

For an $n \times n$ matrix, $M=\left(m_{i, j}\right)$, the permanent of $M$ is defined as

$$
\operatorname{per}(M) \triangleq \sum_{f \in S_{n}} \prod_{i \in[n]} m_{i, f(i)} .
$$

Definition 1. A matrix of particular interest is the Toeplitz matrix $A_{r, n}=\left(a_{i, j}\right)$ of size $n \times n$ defined by

$$
a_{i, j} \triangleq\left\{\begin{array}{ll}
1 & |i-j| \leqslant r \\
0 & \text { otherwise }
\end{array}, \quad i, j \in[n] .\right.
$$

The following lemma is well known [27], [29], [36], [42].

Lemma 2. With the above definitions, it holds that

$$
\left|B_{r, n}\right|=\operatorname{per}\left(A_{r, n}\right) .
$$

Proof: This result follows from

$$
\begin{aligned}
\operatorname{per}\left(A_{r, n}\right) & =\sum_{f \in S_{n}} \prod_{i \in[n]} a_{i, f(i)} \\
& =\left|\left\{f \in S_{n}|\forall i \in[n]:| i-f(i) \mid \leqslant r\right\}\right| \\
& =\left|\left\{f \in S_{n} \mid d\left(\operatorname{Id}_{n}, f\right) \leqslant r\right\}\right| \\
& =\left|B_{r, n}\left(\operatorname{Id}_{n}\right)\right|=\left|B_{r, n}\right| .
\end{aligned}
$$

Note that for any fixed radius $r$, tight asymptotic bounds on $\left|B_{r, n}\right|$ are known [28], [31], [36], [41]. However, in this paper we are interested in the case of radius $r=\rho \cdot(n-1)$, where $\rho \in[0,1]$ is a real constant. This is motivated by the scaling of rank-modulation schemes. Consider flash-memory cells as an example, with fixed minimal and maximal charge levels. When increasing the number of cells, $n$, and assuming a bounded charge level distortion, we obtain a distance (due to distortion) that grows linearly with $n$. Note that in expressions like $r=\rho \cdot(n-1)$ we always implicitly assume that $\rho$ is such that $r$ is an integer, and we shall therefore assume throughout the paper that $\rho$ is in fact rational. We call $\rho$ the normalized radius.

Because of this particular asymptotic setup, $A_{\rho, n}$ and $B_{\rho, n}$ will in the following, with a slight abuse of notation, stand for $A_{\rho \cdot(n-1), n}$ and $B_{\rho \cdot(n-1), n}$, respectively. Moreover, because $\left|B_{\rho, n}\right|=1$ for $\rho=0$ and $\left|B_{\rho, n}\right|=n$ ! for $\rho=1$, i.e., the size of balls for $\rho=0$ and $\rho=1$ are known exactly, in the following we will typically focus on the range $0<\rho<1$ instead of the range $0 \leqslant \rho \leqslant 1$.

We conclude this section by recalling a variety of definitions and results that we will use throughout the paper.

- Stirling's approximation of $n$ ! (see, e.g., [20]) is

$$
n !=\left(\frac{n}{e}\right)^{n} \cdot 2^{o(n)} .
$$

- The binary entropy function is defined to be

$$
h(x) \triangleq-x \cdot \log _{2}(x)-(1-x) \cdot \log _{2}(1-x) .
$$

- The Lambert $W$ function is defined by

$$
z=W(z) \cdot \exp (W(z)) .
$$

(In this paper, $z$ is limited to non-negative real values.)

- A doubly-stochastic matrix is a square $n \times n$ matrix with non-negative real entries for which the sum of each row and each column equals 1 .

- The expression $0 \cdot \log _{2}(0)$ and the expression $0 \cdot \log _{2}\left(\frac{0}{0}\right)$ are both defined to be equal to 0 .

\section{RESULTS}

The main results of this paper are new lower bounds on the size of balls over permutations with the infinity metric. The quality of these new lower bounds is measured by the asymptotic gap between the known upper bounds and the new lower bounds. In this section, we first define what we mean by an asymptotic gap. We then state this gap for known upper and known lower bounds in Section \II-A and for known upper and new lower bounds in Section III-B All derivations for these results will be given in Sections [V] and $\mathrm{V}$

Definition 3. Fix some real constant $\rho \in(0,1)$. Given some upper bound $\Phi$ and some lower bound $\varphi$ on the ball size, i.e.,

$$
\varphi(\rho, n) \leqslant\left|B_{\rho, n}\right| \leqslant \Phi(\rho, n),
$$

where the inequalities are assumed to hold for all positive integers $n$ for which $\rho \cdot(n-1)$ is an integer, we define the asymptotic gap between the upper bound $\Phi$ and the lower bound $\varphi$ to be

$$
\operatorname{Gap}_{\varphi}^{\Phi}(\rho) \triangleq \limsup _{n \rightarrow \infty} \frac{1}{n} \log _{2}\left(\frac{\Phi(\rho, n)}{\varphi(\rho, n)}\right) .
$$

In Section VI-A we will discuss some ball-packing and some Gilbert-Varshamov type bounds (see, e.g., [33, Section 17.7]), both of which depend on the asymptotic size of balls. Clearly, the better we know the asymptotic size of balls, i.e., the smaller the $\operatorname{gap} \operatorname{Gap}_{\varphi}^{\Phi}(\rho)$ is, the stronger the statements will be. 


\section{A. Gap Based on Known Upper and Known Lower Bounds}

Based on a known upper bound $\Phi_{1}$ and a known lower bound $\varphi_{1}$, both detailed in Theorem 8 we obtain the following result.

Theorem 4. It holds that

$$
\operatorname{Gap}_{\varphi_{1}}^{\Phi_{1}}(\rho)=\left\{\begin{array}{cc}
\left(4-2 \log _{2}(e)\right) \cdot \rho & 0<\rho \leqslant \frac{1}{2} \\
2 \cdot(\rho-1) \cdot \log _{2}(e) & \\
-(2 \rho+1) \cdot \log _{2}(\rho) & \frac{1}{2} \leqslant \rho<1
\end{array}\right.
$$

The lower bound of $\varphi_{1}$ was very recently improved, and an asymptotic analysis of this improvement, which we denote $\varphi_{1}^{\prime}$, is given in Theorem 9 This improvement, however, holds only for half the range of $\rho$. By comparing the upper bound of $\Phi_{1}$ and the improved known lower bound $\varphi_{1}^{\prime}$, we obtain the following result.

Theorem 5. For all $0<\rho<\frac{1}{2}$ it holds that

$$
\operatorname{Gap}_{\varphi_{1}^{\prime}}^{\Phi_{1}}(\rho)=2 \cdot\left(h\left(\mu^{*}\right)+\log _{2}\left(\mu^{*}\right)\right) \cdot \rho,
$$

where $\mu^{*}$ is the constant defined by

$$
\mu^{*} \triangleq \frac{1}{1+W\left(e^{-1}\right)} \approx 0.782
$$

The result of Theorems 4 and 5 are visualized by curves (a) and (a'), respectively, in Fig. 1

\section{B. Gap Based on Known Upper and New Lower Bounds}

We present two new lower bounds on the size of balls over permutations with the infinity metric. The first new lower bound, denoted $\varphi_{2}$, is given in Theorem 13 . The other new lower bound, denoted $\varphi_{3}$, is detailed in Theorems 16 and 21 Based on a known upper bound $\Phi_{1}$ and the new lower bounds $\varphi_{2}$ and $\varphi_{3}$, we obtain the following results.

Theorem 6. It holds that

$$
\operatorname{Gap}_{\varphi_{2}}^{\Phi_{1}}(\rho)= \begin{cases}\left(3-2 \log _{2}(e)\right) \cdot \rho & 0<\rho \leqslant \frac{1}{2} \\ 2 \cdot(1-\rho) \cdot\left(1-\rho-\log _{2} e\right) & \\ -2 \rho \cdot \log _{2} \rho & \frac{1}{2} \leqslant \rho<1\end{cases}
$$

Theorem 7. It holds that

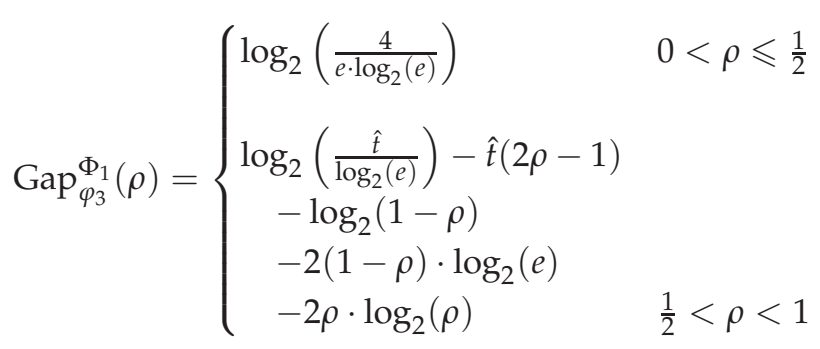

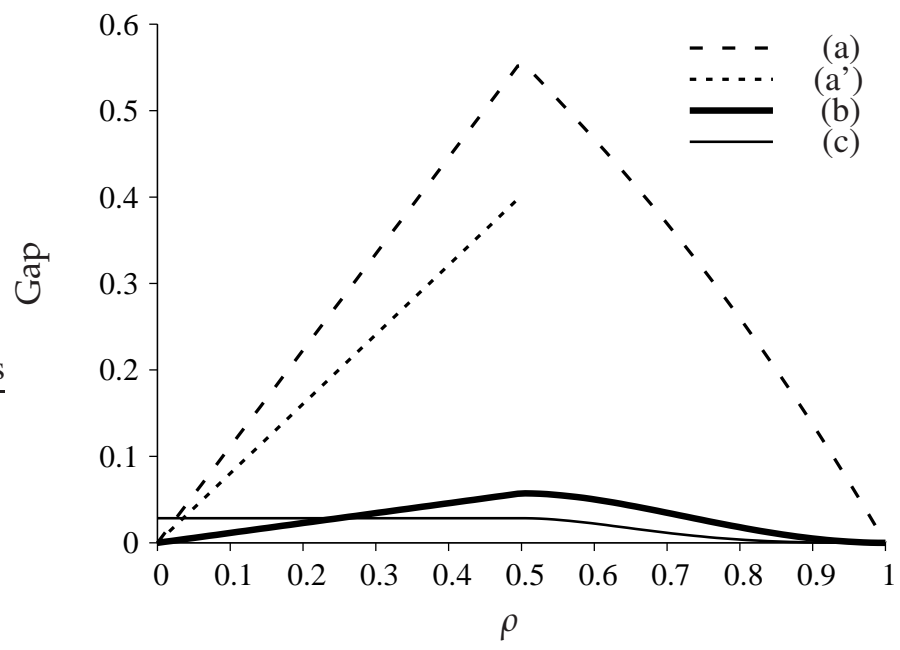

Figure 1. (a) $\operatorname{Gap}_{\varphi_{1}}^{\Phi_{1}}(\rho)$ of Theorem 4 (a') $\operatorname{Gap}_{\varphi_{1}^{\prime}}^{\Phi_{1}}(\rho)$ of Theorem [5. (b) $\operatorname{Gap}_{\varphi_{2}}^{\Phi_{1}}(\rho)$ of Theorem 6 (c) $\operatorname{Gap}_{\varphi_{3}}^{\Phi_{1}}(\rho)$ of Theorem 7

where

$$
\hat{t} \triangleq \log _{2}(e) \cdot\left(\frac{2(1-\rho)}{2 \rho-1}-W\left(\frac{(1-\rho) \cdot \exp \left(\frac{2(1-\rho)}{2 \rho-1}\right)}{2 \rho-1}\right)\right) .
$$

The result of Theorems 6 and 7 are visualized by curves (b) and (c), respectively, in Fig. 1. Note that the curves (b) and (c) cross at

$$
\rho=\frac{\log _{2}\left(\frac{4}{e \cdot \log _{2}(e)}\right)}{3-2 \log _{2}(e)} \approx 0.249 .
$$

\section{AnALysis of KNOWn Bounds}

The following theorems summarize, to the best of our knowledge, the tightest known bounds for balls in $\left(S_{n}, d_{\infty}\right)$.

Theorem 8. It holds that

$$
\varphi_{1}(\rho, n) \leqslant\left|B_{\rho, n}\right| \leqslant \Phi_{1}(\rho, n),
$$

where

$$
\begin{aligned}
& \log _{2} \varphi_{1}(\rho, n)
\end{aligned}
$$

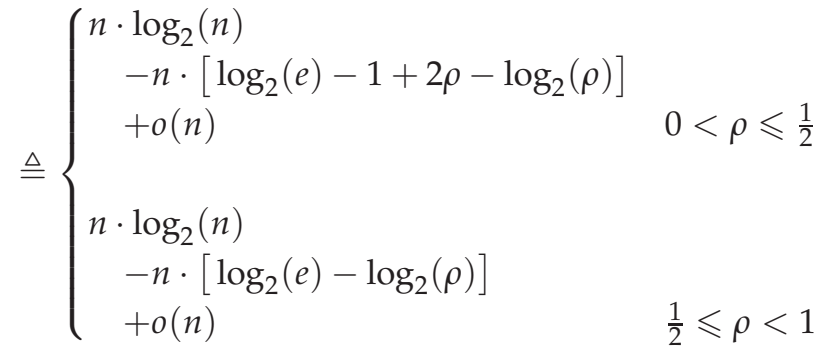


and

$$
\begin{aligned}
& \log _{2} \Phi_{1}(\rho, n) \\
& \triangleq\left\{\begin{array}{l}
n \cdot \log _{2}(n) \\
-n \cdot\left[\left(\log _{2}(e)-1\right) \cdot(2 \rho+1)-\log _{2}(\rho)\right] \\
+o(n) \\
n \cdot \log _{2}(n) \\
-n \cdot\left[\log _{2}(e) \cdot(3-2 \rho)+2 \rho \cdot \log _{2}(\rho)\right] \\
+o(n)
\end{array}\right.
\end{aligned}
$$$$
0<\rho \leqslant \frac{1}{2}
$$$$
\frac{1}{2} \leqslant \rho<1
$$

Proof: These bounds follow from results in [17], [27], [29], [42]. For more details, see Appendix A

Comparing the upper bound $\Phi_{1}$ with the lower bound $\varphi_{1}$, one obtains immediately the result stated in Theorem 4.

Theorem 9. For all $0<\rho \leqslant \frac{1}{2}$, it holds that

$$
\left|B_{\rho, n}\right| \geqslant \varphi_{1}^{\prime}(\rho, n),
$$

where

$$
\begin{aligned}
\log _{2} \varphi_{1}^{\prime}(\rho, n)=n & \cdot \log _{2} n \\
& -n \cdot\left[\left(\log _{2}(e)-1\right) \cdot(2 \rho+1)-\log _{2}(\rho)\right. \\
& \left.\quad+2 \cdot\left(h\left(\mu^{*}\right)+\log _{2}\left(\mu^{*}\right)\right) \cdot \rho\right] \\
& +o(n),
\end{aligned}
$$

and where $\mu^{*}$ is the constant defined by

$$
\mu^{*} \triangleq \frac{1}{1+W\left(e^{-1}\right)} \approx 0.782
$$

Proof: This result follows from an asymptotic analysis of the conjectured lower bound in [29], which was very recently proven in [21]. The asymptotic analysis is briefly sketched in Appendix B

\section{NEW LOWER BOUNDS}

In this section we present new lower bounds on the size of balls in $\left(S_{n}, d_{\infty}\right)$. These lower bounds are based on the following theorem, which is a variant of a result in [32]. The art in using this theorem is to find $Q$ matrices that yield large right-hand sides in (2) and that are analytically tractable.

Theorem 10. Let $M \triangleq\left(m_{i, j}\right)$ be an $n \times n$ matrix with nonnegative entries and $\operatorname{per}(M)>0$, and let $Q \triangleq\left(q_{i, j}\right)$ be an $n \times n$ doubly-stochastic matrix such that $q_{i, j}=0$ whenever $m_{i, j}=0$. Then

$$
\log _{2} \operatorname{per}(M) \geqslant \log _{2}\left(\frac{n !}{n^{n}}\right)+\sum_{i, j \in[n]}\left(-q_{i, j} \log _{2} \frac{q_{i, j}}{m_{i, j}}\right) .
$$

Proof: Let $\varepsilon>0$ and let $M^{(\varepsilon)} \triangleq\left(m_{i, j}^{(\varepsilon)}\right)$ be the matrix that is obtained from $M$ by replacing zeros by $\varepsilon$. Because $M^{(\varepsilon)}$ contains only strictly positive entries, it follows from a theorem by Sinkhorn [40] that there exist two diagonal matrices $D$ and
$D^{\prime}$ with positive diagonal elements such that $D \cdot M^{(\varepsilon)} \cdot D^{\prime}$ is a doubly-stochastic matrix. Let $D$ and $D^{\prime}$ be given by

$$
\begin{aligned}
D & \triangleq \operatorname{diag}\left(d_{1}, \ldots, d_{n}\right), \\
D^{\prime} & \triangleq \operatorname{diag}\left(d_{1}^{\prime}, \ldots, d_{n}^{\prime}\right),
\end{aligned}
$$

where $d_{i}, i \in[n]$, and $d_{j}^{\prime}, j \in[n]$, are positive real numbers. Note that the element in the $i$-th row and the $j$-th column of $D \cdot M^{(\varepsilon)} \cdot D^{\prime}$ is given by $d_{i} \cdot m_{i, j}^{(\varepsilon)} \cdot d_{j}^{\prime}$. Then

$$
\begin{aligned}
& \log _{2} \operatorname{per}\left(M^{(\varepsilon)}\right) \\
& \stackrel{(\mathrm{a})}{=} \log _{2} \operatorname{per}\left(D \cdot M^{(\varepsilon)} \cdot D^{\prime}\right)-\sum_{i \in[n]} \log _{2}\left(d_{i}\right)-\sum_{j \in[n]} \log _{2}\left(d_{j}^{\prime}\right)
\end{aligned}
$$

$$
\begin{aligned}
& \stackrel{(\text { b) }}{\geqslant} \log _{2}\left(\frac{n !}{n^{n}}\right)-\sum_{i \in[n]} \log _{2}\left(d_{i}\right)-\sum_{j \in[n]} \log _{2}\left(d_{j}^{\prime}\right) \\
& \stackrel{\text { (c) }}{\geqslant} \log _{2}\left(\frac{n !}{n^{n}}\right)-\sum_{i \in[n]} \log _{2}\left(d_{i}\right)-\sum_{j \in[n]} \log _{2}\left(d_{j}^{\prime}\right) \\
& -\sum_{i, j \in[n]} q_{i, j} \cdot \log _{2}\left(\frac{q_{i, j}}{d_{i} \cdot m_{i, j}^{(\varepsilon)} \cdot d_{j}^{\prime}}\right) \\
& =\log _{2}\left(\frac{n !}{n^{n}}\right)-\sum_{i, j \in[n]} q_{i, j} \cdot \log _{2}\left(\frac{q_{i, j}}{m_{i, j}^{(\varepsilon)}}\right) \\
& \stackrel{\text { (d) }}{\geqslant} \log _{2}\left(\frac{n !}{n^{n}}\right)-\sum_{i, j \in[n]} q_{i, j} \cdot \log _{2}\left(\frac{q_{i, j}}{m_{i, j}}\right) \text {. }
\end{aligned}
$$

We justify the steps taken: Step (a) follows by noting that $M^{(\varepsilon)}$ is obtained from $D \cdot M^{(\varepsilon)} \cdot D^{\prime}$ by factoring out $d_{i}$ from the $i$ th row and $d_{j}^{\prime}$ from the $j$ th column, for all $i, j \in[n]$. Step (b) follows from Van der Waerden's conjecture (proven by Falikman [16] and by Egorychev [15]), which states that for any $n \times n$ doubly-stochastic matrix $U$, we have $\operatorname{per}(U) \geqslant$ $n ! / n^{n}$. Step (c) follows from the non-negativity of relative entropy. Finally, Step (d) follows from $m_{i, j}^{(\varepsilon)} \geqslant m_{i, j}$ for all $i, j \in[n]$, and by noting that we require $q_{i, j}=0$ whenever $m_{i, j}=0$.

Note that $\lim _{\varepsilon \rightarrow 0} \operatorname{per}\left(M^{(\varepsilon)}\right)=\operatorname{per}(M)$, because the permanent of a matrix is a multilinear function of the entries of the matrix. With this, applying the limit $\varepsilon \rightarrow 0$ to the expression in (3), we obtain (2).

We note that $D$ and $D^{\prime}$ are auxiliary matrices in the proof of Theorem 10. Only their existence matters, while their entries do not play a role in (2). For matrices $M$ with strictly positive entries (and possibly some other classes of matrices), the righthand side of (2) can be maximized with the help of Sinkhorn's balancing algorithm [40], see, e.g., the discussions in [23], [32], 45] 1 ]

In the following, we will apply Theorem 10 with $M=$ $A_{r, n}$ and with two classes of $Q$ matrices. The first class of $Q$ matrices will ultimately yield Theorem 6 , whereas the second class of $Q$ matrices will ultimately yield Theorem 7 .

\footnotetext{
${ }^{1}$ Strictly speaking, we do not need Sinkhorn's balancing algorithm to prove our results. However, we mention this algorithm here because it played a key role for analyzing setups with finite $n$ and $r$ and coming up with the $Q_{r, n}$ matrices in Definitions 14 and 17 for general $n$ and $r$.
} 


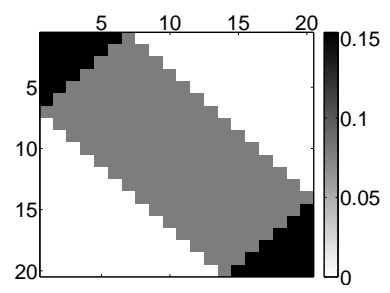

(a) $Q_{6,20}$ of Def. 11

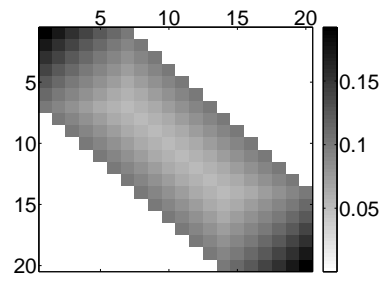

(c) $Q_{6,20}$ of Def. 14

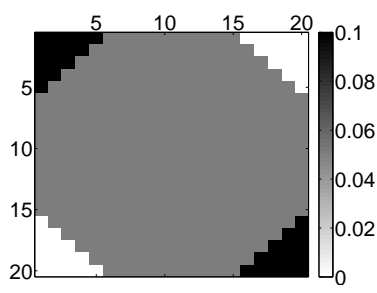

(b) $Q_{14,20}$ of Def. 11

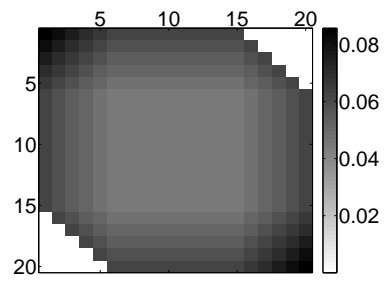

(d) $Q_{14,20}$ of Def. 17
Figure 2. Matrices $Q_{r, n}$ used in this paper to obtain various lower bounds. Note that for every instance, the support of $Q_{r, n}$ equals the support of $A_{r, n}$.

\section{A. First Class of $Q$ Matrices}

Definition 11. For $0 \leqslant r \leqslant \frac{n-1}{2}$, we define the matrix $Q_{r, n}=$ $\left(q_{i, j}\right)$ with entries

$$
q_{i, j} \triangleq\left\{\begin{array}{ll}
\frac{2}{2 r+1} & i+j \leqslant r+1 \text { or } i+j \geqslant 2 n-r+1 \\
\frac{a_{i, j}}{2 r+1} & \text { otherwise }
\end{array} .\right.
$$

For $\frac{n-1}{2} \leqslant r \leqslant n-1$, we define the matrix $Q_{r, n}=\left(q_{i, j}\right)$ with entries

$$
q_{i, j} \triangleq \begin{cases}\frac{2}{n} & i+j \leqslant n-r \text { or } i+j \geqslant n+r+2 \\ \frac{a_{i, j}}{n} & \text { otherwise. }\end{cases}
$$

Lemma 12. The matrix $Q_{r, n}$ in Definition 11 is a doublystochastic matrix with the same support as $A_{r, n}$.

\section{Proof: See Appendix C}

For $n=20$ and $r=6$, the resulting $Q_{r, n}$ matrix is depicted in Fig. 2(a), whereas for $n=20$ and $r=14$, the resulting $Q_{r, n}$ matrix is depicted in Fig. 2(b).

Theorem 13. Fix some $\rho, 0<\rho<1$. It holds that

$$
\left|B_{\rho, n}\right| \geqslant \varphi_{2}(\rho, n),
$$

where we define

$$
\begin{aligned}
& \log _{2} \varphi_{2}(\rho, n)
\end{aligned}
$$

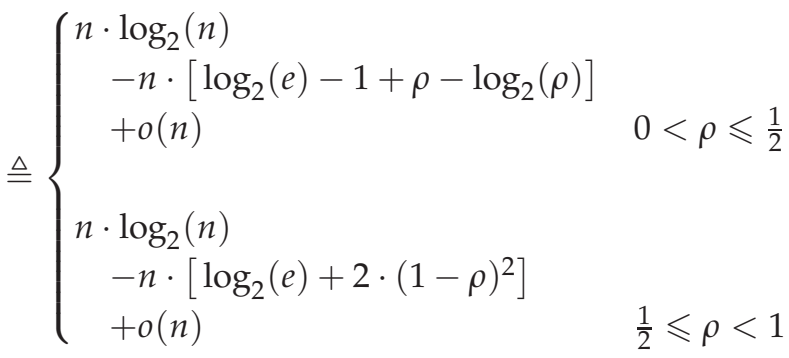

Proof: See Appendix D.
Comparing the upper bound $\Phi_{1}$ with the new lower bound $\varphi_{2}$, one obtains immediately the result stated in Theorem 6

We conclude this section with several comments on the matrices $Q_{r, n}$. The matrix $Q_{r, n}$ that we defined in (4) already appeared in [21], [29], and that of (5] already appeared in [17]. Although [17], [21], [29] introduce the same matrices, they consider a different approach to obtain a lower bound on $\operatorname{per}\left(A_{r, n}\right)$ than the one presented in this paper, and with that they obtain a different lower bound on $\left|B_{\rho, n}\right|$.

We also note that the definition of the matrix $Q_{r, n}$ in (4) works not only for the range $0<\rho \leqslant \frac{1}{2}$, but for the entire range $0<\rho<1$. However, we define these matrices only for the range $0<\rho \leqslant \frac{1}{2}$, because they yield a weaker lower bound than the lower bound in Theorem 13 for the range $\frac{1}{2} \leqslant \rho<1$.

\section{B. Second Class of $Q$ Matrices}

Our second class for the $Q$ matrices are more sophisticated than our first class. In the following, we will separately discuss the cases $0<\rho \leqslant 1 / 2$ and $\frac{1}{2}<\rho<1$. Note that the $Q$ matrix that we will use for the case $\frac{1}{2}<\rho<1$ maximizes the right-hand side of (2) for $M=A_{r, n}$. This is in contrast to the $Q$ matrix that we will use for the case $0<\rho \leqslant 1 / 2$. This $Q$ matrix does not, in general, maximize the right-hand side of (2) for $M=A_{r, n}$. An exception is the case where $n$ is even and $r=\frac{n-2}{2}$.

1) Range $0<\rho \leqslant \frac{1}{2}$ : We start our discussion of this case with the following definition.

Definition 14. Fix some $r, 1 \leqslant r \leqslant \frac{n-2}{2}$. Let $Q_{r, n} \triangleq\left(q_{i, j}\right)$ be the $n \times n$-matrix with entries

$$
q_{i, j} \triangleq a_{i, j} \cdot C \cdot \tilde{q}_{i, j}
$$

where

$$
\tilde{q}_{i, j}= \begin{cases}\alpha_{r}^{(r+1-i)+(r+1-j)} & 1 \leqslant i \leqslant r+1,1 \leqslant j \leqslant r+1 \\ \alpha_{r}^{i-(n-r)+j-(n-r)} & n-r \leqslant i \leqslant n, n-r \leqslant j \leqslant n \\ \alpha_{r}^{|i-j|} & \text { otherwise }\end{cases}
$$

where $C$ is given by

$$
C \triangleq\left(\alpha_{r}-1\right) \cdot \alpha_{r}^{-r-1}=\frac{\alpha_{r}-1}{\alpha_{r}+1}
$$

and where $\alpha_{r}>0$ satisfies

$$
\alpha_{r}^{r+1}-\alpha_{r}-1=0 .
$$

Note that the second expression for $C$ in (6) follows from the first expression for $C$ in (6), along with (7). Moreover, note that the $\alpha_{r}>0$ satisfying (7) is unique. (This can be proven by analyzing the function $\alpha \mapsto \alpha^{r+1}-\alpha-1$.)

For $n=20$ and $r=6$, the resulting $Q_{r, n}$ matrix is depicted in Fig. 2(c).

Lemma 15. The matrix $Q_{r, n}$ in Definition 14 is a doublystochastic matrix with the same support as $A_{r, n}$.

Proof: See Appendix E 
Theorem 16. Fix some $\rho, 0<\rho \leqslant \frac{1}{2}$. It holds that

$$
\left|B_{\rho, n}\right| \geqslant \varphi_{3}(\rho, n),
$$

where we define

$$
\begin{aligned}
& \log _{2} \varphi_{3}(\rho, n) \\
& \triangleq n \cdot \log _{2}(n) \\
& \quad-n \cdot\left[\left(\log _{2}(e)-1\right) \cdot 2 \rho-\log _{2}(\rho)-\log _{2}\left(\log _{2}(e)\right)+1\right] \\
& \quad+o(n) .
\end{aligned}
$$

Proof: See Appendix E]

Comparing the upper bound $\Phi_{1}$ with the new lower bound $\varphi_{3}$, one obtains immediately the result stated in Theorem 7 for the case $0<\rho \leqslant \frac{1}{2}$.

2) Range $\frac{1}{2}<\rho<1$ : We start our discussion of this case with the following definition.

Definition 17. Fix some $r, \frac{n-1}{2}<r<n-1$. Let $Q_{r, n} \triangleq\left(q_{i, j}\right)$ be the $n \times n$-matrix with entries

$$
q_{i, j}=a_{i, j} \cdot C \cdot \exp _{2}\left(\lambda_{i}\right) \cdot \exp _{2}\left(\lambda_{j}^{\prime}\right), \quad i, j \in[n],
$$

where

$$
\begin{aligned}
& \lambda_{i} \triangleq \begin{cases}((n-r)-i) \cdot \log _{2}\left(\alpha_{r, n}\right) & 1 \leqslant i \leqslant n-r \\
0 & n-r \leqslant i \leqslant r+1 \\
(i-(r+1)) \cdot \log _{2}\left(\alpha_{r, n}\right) & r+1 \leqslant i \leqslant n\end{cases} \\
& \lambda_{j}^{\prime} \triangleq \lambda_{j}, \quad j \in[n], \\
& C \triangleq\left(\alpha_{r, n}-1\right) \cdot \alpha_{r, n}^{-(n-r)} \\
& =\frac{\alpha_{r, n}-1}{(2 r-n+2)-(2 r-n) \cdot \alpha_{r, n}},
\end{aligned}
$$

where $\alpha_{r, n}>0$ satisfies

$$
\alpha_{r, n}^{n-r}+(2 r-n) \cdot \alpha_{r, n}-(2 r-n+2)=0 .
$$

We note that

- $\lambda_{n+1-i}=\lambda_{i}, i \in[n]$,

- $\lambda_{n+1-j}^{\prime}=\lambda_{j}^{\prime}, j \in[n]$.

Additionally, we observe that the second expression for $C$ in (10) follows from the first expression for $C$ in (9), along with (11). Moreover, note that the $\alpha_{r, n}>0$ satisfying (11) is unique. (This can be proven by analyzing the function $\alpha \mapsto \alpha^{n-r}+(2 r-n) \cdot \alpha-(2 r-n+2)$.)

For $n=20$ and $r=14$, the resulting $Q_{r, n}$ matrix is depicted in Fig. 2(d).

Lemma 18. The matrix $Q_{r, n}$ in Definition 17 is a doublystochastic matrix with the same support as $A_{r, n}$.

\section{Proof: See Appendix G}

Lemma 19. Fix some $r, \frac{n-1}{2}<r<n-1$. It holds that

$$
\begin{aligned}
\log _{2}\left|B_{r, n}\right| \geqslant & \log _{2}(n !)-n \log _{2}(n)-n \cdot \log _{2}\left(\alpha_{r, n}-1\right) \\
& +(n-r) \cdot(2 r-n+2) \cdot \log _{2}\left(\alpha_{r, n}\right),
\end{aligned}
$$

where $\alpha_{r, n}$ was specified in 11.

\section{Proof: See Appendix $\mathrm{H}$}

Note that the lower bound in Lemma 19 contains the constant $\alpha_{r, n}$. In order to get rid of this constant, the upcoming Lemma 20 suitably approximates this constant and Theorem 21 will then show the updated expression for the lower bound.

Lemma 20. Fix some $\rho$ with $\frac{1}{2}<\rho<1$. Let $r \triangleq \rho \cdot(n-1)$. Then $\alpha_{r, n}$ from (11) satisfies

$$
\alpha_{r, n}=1+\left(\hat{t}+\Theta\left(n^{-1}\right)\right) \cdot\left(2^{\frac{1}{(n-1)(1-\rho)+1}}-1\right)
$$

where

$$
\hat{t} \triangleq \log _{2}(e) \cdot\left(\frac{2(1-\rho)}{2 \rho-1}-W\left(\frac{(1-\rho) \cdot \exp \left(\frac{2(1-\rho)}{2 \rho-1}\right)}{2 \rho-1}\right)\right) .
$$

Proof: See Appendix 凹

Theorem 21. Fix some $\rho$ with $\frac{1}{2}<\rho<1$ a constant. Let $r \triangleq \rho \cdot(n-1)$. It holds that

$$
\left|B_{r, n}\right| \geqslant \varphi_{3}(\rho, n),
$$

where

$$
\begin{aligned}
\log _{2} & \varphi_{3}(\rho, n) \\
\triangleq & n \cdot \log _{2}(n) \\
& -n \cdot\left[\log _{2}\left(\frac{e \cdot \hat{t}}{\log _{2}(e)}\right)-\hat{t} \cdot(2 \rho-1)-\log _{2}(1-\rho)\right] \\
& +o(n),
\end{aligned}
$$

and where $\hat{t}$ is given by (12).

\section{Proof: See Appendix J}

Comparing the upper bound $\Phi_{1}$ with the new lower bound $\varphi_{3}$, one obtains immediately the result stated in Theorem 7 for the case $\frac{1}{2}<\rho<1$.

\section{CONCLUSION AND OUTLOOK}

We conclude this paper by commenting about the newly obtained lower bounds and by stating some open problems.

\section{A. Implications of the New Bounds}

Previous works on error-correcting codes over permutations with the infinity norm [30], [42], [48] used bounds on the size of balls in this metric to state ball-packing and GilbertVarshamov-like bounds. Since in this paper we improved the lower bound on the size of balls, these new bounds affect the ball-packing bound (stated in [42]). If we consider errorcorrecting codes in $S_{n}$ of rate $R$ and normalized distance $\delta$, the ball-packing bound 2 states that

$$
2^{R n} \leqslant \frac{n !}{\mid B_{\lfloor(\delta(n-1)-1) / 2\rfloor, n \mid},}
$$

${ }^{2}$ We comment that in [42], $\delta$ was defined in a slightly different manner, by $\delta \triangleq d / n$, where $d$ is the minimum distance of the code. We instead use $\delta \triangleq d /(n-1)$, to be consistent with the normalization used throughout the paper. The change due to the difference in normalization is subsumed in the $o(1)$ additive factor in the upper bound on the rate. 
or in asymptotic form,

$$
R \leqslant \log _{2} n-\log _{2} e-\frac{1}{n} \log _{2}\left|B_{\delta / 2, n}\right|+o(1) .
$$

Using the bounds on $\left|B_{\delta / 2, n}\right|$ known at that time, it was stated in [42, Th. 27] that

$$
R \leqslant \delta+\log _{2} \frac{1}{\delta}+o(1) .
$$

However, now we can use the improved lower bounds $\varphi_{2}$ and $\varphi_{3}$, and obtain a stronger asymptotic form for the ball-packing bound,

$$
R \leqslant \begin{cases}\frac{\delta}{2}+\log _{2} \frac{1}{\delta}+o(1) & 0 \leqslant \frac{\delta}{2} \leqslant \xi \\ \left(\log _{2}(e)-1\right)(\delta-1)+\log _{2} \frac{1}{\delta}+1 & \\ -\log _{2}\left(\log _{2}(e)\right)+o(1) & \xi \leqslant \frac{\delta}{2} \leqslant \frac{1}{2},\end{cases}
$$

where $\xi \triangleq \frac{2-\log _{2}(e)-\log _{2}\left(\log _{2}(e)\right)}{3-2 \log _{2}(e)} \approx 0.249$ is the crossover point of curves (b) and (c) in Fig. 11 The ball-packing bound for error-correcting codes is shown in Fig. 3. before and after the improvement of this paper. We do note that while the improvement in the bound is substantial, it is still weaker than the code-anticode bound described in [42]. (However, the bound in [42] does not have the geometric interpretation of packing balls.)

In contrast, in the case of covering codes over permutations with the infinity norm, the new bounds in this paper do improve the best upper bounds on the rate of the codes. Let us now consider covering codes in $S_{n}$ of rate $R$ and normalized covering radius $\rho$. The upper bound of [17, Th. 3] is

$$
2^{R n} \leqslant \frac{n !(1+\ln (n !))}{\left|B_{\rho, n}\right|} .
$$

Using the asymptotic bounds on $\left|B_{\rho, n}\right|$ known at that time, an asymptotic form was given in [17, Th. 15] as

$$
R \leqslant \begin{cases}2 \rho+\log _{2} \frac{1}{\rho}+o(1) & 0 \leqslant \rho \leqslant \frac{1}{2} \\ 2(1-\rho)+o(1) & \frac{1}{2} \leqslant \rho \leqslant 1 .\end{cases}
$$

Now, using $\varphi_{2}$ and $\varphi_{3}$, we can state an improved upper bound

$$
R \leqslant \begin{cases}\rho-1+\log _{2} \frac{1}{\rho}+o(1) & 0 \leqslant \rho \leqslant \xi \\ (2 \rho-1)\left(\log _{2}(e)-1\right) & \\ \quad+\log _{2} \frac{1}{\rho}+\log _{2}\left(\log _{2}(e)\right) & \xi \leqslant \rho \leqslant \frac{1}{2} \\ \log _{2}(\hat{t})-\log _{2}\left(\log _{2}(e)\right) & \\ \quad-(2 \rho-1) \hat{t}-\log _{2}(1-\rho) & \frac{1}{2} \leqslant \rho \leqslant 1,\end{cases}
$$

where $\xi$ as defined above, and $\hat{t}$ as defined in Theorem 7

We observe that the largest improvement in Fig. 1 (between the previous lower bounds and our new lower bounds) occurs at $\rho=\frac{1}{2}$. This manifests in Fig. 4 in a similar manner, showing the largest improvement between curves (b) and (c) occurring at $\rho=\frac{1}{2}$. However, in Fig. 3, the largest improvement between curves (b) and (c) occurs at $\delta=1$. This is due to the fact that the ball-packing bound uses $\left|B_{\delta / 2, n}\right|$.

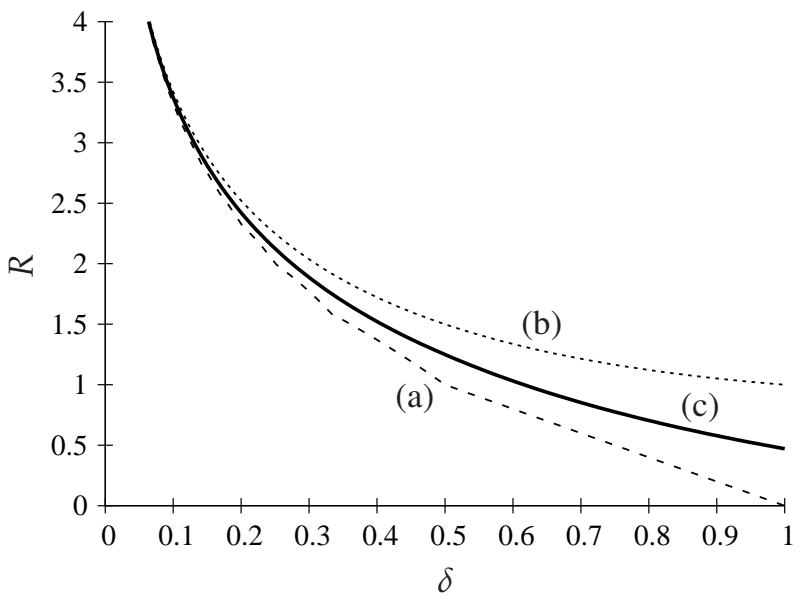

Figure 3. Upper bounds on the size of error-correcting codes over permutations with the infinity norm (rate $R$ as a function of the normalized minimum distance $\delta$ ): (a) The code-anticode bound of [42]; (b) The ballpacking bound of [42]; (c) The ball-packing bound using the new bounds of this paper.

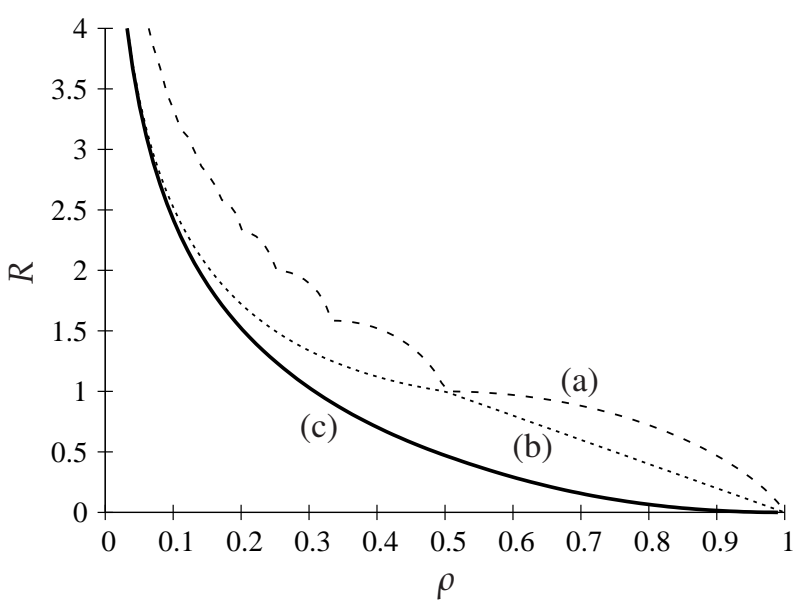

Figure 4. Upper bounds on the size of optimal covering codes over permutations with the infinity norm (rate $R$ as a function of the normalized covering radius $\rho$ ): (a) The covering-code construction of [17; (b) The upper bound of [17]; (c) The upper bound using the new bounds of this paper.

\section{B. Open Problems}

We now turn to discuss some open problems. As mentioned at the beginning of Section $\mathrm{V}-\mathrm{B}$, the $Q$ matrix that we use there for the case $\frac{1}{2}<\rho<1$ maximizes the right-hand side of (2) for $M=A_{r, n}$. This is in contrast to the $Q$ matrix that we use for the case $0<\rho \leqslant 1 / 2$, which in general does not maximize the right-hand side of (2) for $M=A_{r, n}$. We leave it as an open problem to find the $Q$ matrix that maximizes the right-hand side of (2) for the case $0<\rho \leqslant 1 / 2$. (So far, analytical considerations, along with some numerical evidence for somewhat small choices of $n$ and $r$, have not led to a closed-form expression for the optimal $Q$ matrix.)

We mention another open problem. Instead of Theorem 10 one can also use the following approach to obtain a lower bound on $\log _{2} \operatorname{per}(M)$. Namely, let $\operatorname{per}_{B}(M)$ be the Bethe permanent of an $n \times n$-matrix with non-negative entries [44]. The following theorem is due to Gurvits [22]. (See also the 
discussion in [44].)

Theorem 22. Let $M \triangleq\left(m_{i, j}\right)$ be an $n \times n$ matrix with nonnegative entries and $\operatorname{per}(M)>0$. Let $Q_{r, n} \triangleq\left(q_{i, j}\right)$ be any $n \times n$ doubly-stochastic matrix such that $q_{i, j}=0$ whenever $m_{i, j}=0$. Then

$$
\begin{aligned}
& \log _{2} \operatorname{per}(M) \\
& \quad \geqslant \log _{2} \operatorname{per}_{\mathrm{B}}(M) \\
& \quad \geqslant \sum_{i, j \in[n]}\left(-q_{i, j} \log _{2}\left(\frac{q_{i, j}}{m_{i, j}}\right)+\left(1-q_{i, j}\right) \log _{2}\left(1-q_{i, j}\right)\right) .
\end{aligned}
$$

For the asymptotic setup of interest in this paper, i.e., $r \triangleq \rho \cdot(n-1)$, and the $Q$-matrices which were defined in Sections $\mathrm{V}-\mathrm{A}$ and $\mathrm{V}-\mathrm{B}$, it turns out that Theorems 10 and 22 lead to the same lower bounds (modulo $o(n)$ terms) except for the boundary case $\rho=0$. It is conceivable that an optimal choice of a $Q$-matrix for Theorem 22 may result in a lower bound that outperforms the bounds obtained in this paper. We leave it as an open problem to analytically find the $Q$ matrix which maximizes the right-hand side of the expression in Theorem 22 for $M=A_{r, n}$.

\section{ACKNOWLEDGMENTS}

The authors would like to thank the associate editor and the anonymous reviewers, whose comments helped to improve the presentation of this paper.

\section{APPENDIX A \\ PROOF OF THEOREM 8}

To the best of our knowledge, the tightest known bounds for balls in $\left(S_{n}, d_{\infty}\right)$ are as follows:

$$
\varphi_{1}(\rho, n) \leqslant\left|B_{\rho, n}\right| \leqslant \Phi_{1}(\rho, n),
$$

where

$$
\begin{aligned}
\varphi_{1}(\rho, n) \triangleq\left\{\begin{array}{ll}
\frac{n ! \cdot(2 r+1)^{n}}{2^{2 r} \cdot n^{n}} & 0<\rho \leqslant \frac{1}{2} \\
\frac{n ! \cdot(2 r+1)^{n}}{2^{n} \cdot n^{n}} & \frac{1}{2} \leqslant \rho<1
\end{array},\right. \\
\Phi_{1}(\rho, n) \triangleq \begin{cases}((2 r+1) !)^{\frac{n-2 r}{2 r+1}} \prod_{i=r+1}^{2 r}(i !)^{\frac{2}{i}} & 0<\rho \leqslant \frac{1}{2} \\
(n !)^{\frac{2 r+2-n}{n}} \prod_{i=r+1}^{n-1}(i !)^{\frac{2}{i}} & \frac{1}{2} \leqslant \rho<1\end{cases}
\end{aligned}
$$

where $r \triangleq \rho \cdot(n-1)$. These bounds are a consequence of the following results:

- For the range $0<\rho \leqslant \frac{1}{2}$, the upper bound was given in [27, Eq. (4)], and the lower bound was given in [29. Eq. (3) and (5)].

- For the range $\frac{1}{2} \leqslant \rho<1$, the upper bound was given in [42, Lemma 25].

- For the range $\frac{1}{2} \leqslant \rho<1$, a slightly weaker lower bound was given in [17, Lemma 14]. However, the bound we cite here, though never presented explicitly, may be deduced from [29, Eq. (3) and (5)] while noting that (in the notation of [29])

$$
\begin{aligned}
\operatorname{per}\left(A^{(r, n)}\right) & =\frac{1}{2^{n}} \cdot \operatorname{per}\left(2 A^{(r, n)}\right) \\
& \geqslant \frac{1}{2^{n}} \cdot \operatorname{per}\left(B^{(r, n)}\right) \\
& \geqslant \frac{n ! \cdot(2 r+1)^{n}}{2^{n} \cdot n^{n}} .
\end{aligned}
$$

We would now like to convert these bounds to a more pleasing asymptotic form. We start with the range $0<\rho \leqslant \frac{1}{2}$. For $\varphi_{1}(\rho, n)$ we obtain

$$
\varphi_{1}(\rho, n)=\frac{n ! \cdot(2 \rho \cdot(n-1)+1)^{n}}{2^{2 \rho \cdot(n-1)} n^{n}}=\frac{(2 \rho n)^{n}}{2^{2 \rho n} e^{n}} \cdot 2^{o(n)},
$$

where we used Stirling's approximation.

On the other hand, the expression for $\Phi_{1}(\rho, n)$ follows from

$$
\begin{aligned}
\Phi_{1}(\rho, n)= & ((2 \rho(n-1)+1) !)^{\frac{n-2 \rho \cdot(n-1)}{2 \rho \cdot(n-1)+1}} \cdot \prod_{i=\rho \cdot(n-1)+1}^{2 \rho \cdot(n-1)}(i !)^{\frac{2}{i}} \\
= & \left(\frac{2 \rho n}{e}\right)^{n(1-2 \rho)} \cdot\left(\prod_{i=\rho \cdot(n-1)+1}^{2 \rho \cdot(n-1)}(i !)^{\frac{2}{i}}\right) \cdot 2^{o(n)} \\
= & \left(\frac{2 \rho n}{e}\right)^{n(1-2 \rho)} \\
& \cdot\left(\prod_{i=\rho \cdot(n-1)+1}^{2 \rho \cdot(n-1)}\left(\left(\frac{i}{e}\right)^{2} 2^{o(1)}\right)\right) \cdot 2^{o(n)} \\
= & \left(\frac{2 \rho n}{e}\right)^{n(1-2 \rho)} \\
& \cdot \frac{1}{e^{2 \rho \cdot(n-1)}} \cdot\left(\frac{(2 \rho \cdot(n-1)) !}{(\rho \cdot(n-1)) !}\right)^{2} \cdot 2^{o(n)} \\
= & \left(\frac{2 \rho n}{e}\right)^{n(1-2 \rho)} \cdot \frac{1}{e^{2 \rho n}} \cdot 2^{4 \rho n} \cdot\left(\frac{\rho n}{e}\right)^{2 \rho n} \cdot 2^{o(n)} \\
= & \left(\frac{2 \rho n}{e}\right)^{n} \cdot\left(\frac{2}{e}\right)^{2 \rho n} \cdot 2^{o(n)},
\end{aligned}
$$

where we used Stirling's approximation whenever a factorial appears. The case of $\frac{1}{2} \leqslant \rho<1$ is handled analogously. Namely, after following similar steps we get

$$
\begin{aligned}
\varphi_{1}(\rho, n) & =\frac{(2 \rho n)^{n}}{2^{n} e^{n}} \cdot 2^{o(n)}, \\
\Phi_{1}(\rho, n) & =\frac{n^{n}}{e^{n(3-2 \rho)} \rho^{2 \rho n}} \cdot 2^{o(n)} .
\end{aligned}
$$

Clearly, the obtained expressions for $\Phi_{1}$ and $\varphi_{1}$ imply the expressions given in the theorem statement.

\section{APPENDIX B}

Proof of Theorem 9

We recall that the asymptotic regime of interest to us is $r=\rho \cdot(n-1)$ for some fixed $\rho$. The conjecture of [29], proven in [21], asserts that for $0<\rho \leqslant \frac{1}{2}$,

$$
\left|B_{\rho, n}\right|>\frac{\sqrt{2 \pi(n+2 r)}}{\omega_{r}^{2}} \cdot\left(\frac{2 r+1}{e}\right)^{n},
$$


where

$$
\omega_{r} \triangleq \frac{\Omega_{r} \cdot e^{r}}{(2 r+1)^{r}}
$$

and

$$
\Omega_{r} \triangleq \sum_{m=0}^{r}\left(\begin{array}{c}
r \\
m
\end{array}\right) \cdot(m+1)^{r}
$$

In order to complete the analysis, we need to find an asymptotic expression for $\Omega_{r}$. To that end, let us denote

$$
\tilde{\Omega}_{r} \triangleq \max \left\{\left(\begin{array}{c}
r \\
m
\end{array}\right) \cdot(m+1)^{r} \mid 0 \leqslant m \leqslant r\right\} .
$$

Then

$$
\tilde{\Omega}_{r} \leqslant \Omega_{r} \leqslant(r+1) \cdot \tilde{\Omega}_{r} .
$$

In the limit $n \rightarrow \infty$, these inequalities imply

$$
\log _{2}\left(\Omega_{r}\right)=\log _{2}\left(\tilde{\Omega}_{r}\right)+o(n) .
$$

In order to find $\tilde{\Omega}_{r}$, we look for the maximal summand in the definition of $\Omega_{r}$. We do so by looking at the ratio of two successive summands,

$$
\frac{\left(\begin{array}{c}
r \\
m
\end{array}\right) \cdot(m+1)^{r}}{\left(\begin{array}{c}
r \\
m-1
\end{array}\right) \cdot m^{r}}
$$

and note that this ratio is monotone decreasing in $m$. We denote $m=\mu \cdot r$ for some suitable $\mu$, and thus we would like to find the value of $\mu$ for which

$$
\frac{\left(\begin{array}{c}
r \\
\mu r
\end{array}\right) \cdot(\mu r+1)^{r}}{\left(\begin{array}{c}
r \\
\mu r-1
\end{array}\right) \cdot(\mu r)^{r}}=1 .
$$

Since $r$ tends to infinity, in the limit, this equation becomes

$$
\frac{1-\mu}{\mu} \cdot e^{1 / \mu}=1
$$

whose exact solution is

$$
\mu=\frac{1}{1+W\left(e^{-1}\right)} \triangleq \mu^{*} .
$$

Thus the value of $m$ we are looking for is

$$
m=\mu^{*} \cdot r \cdot(1+o(1)),
$$

and so

$$
\begin{aligned}
\log _{2}\left(\Omega_{r}\right) & =\log _{2}\left(\tilde{\Omega}_{r}\right)+o(n) \\
& =n \cdot\left(h\left(\mu^{*}\right)+\log _{2}\left(\mu^{*} \rho n\right)\right) \cdot \rho+o(n) .
\end{aligned}
$$

Plugging this back into (13) and (14), we obtain the promised result.

\section{APPENDIX C \\ Proof of LemMa 12}

We begin by noting that the support of $Q_{r, n}$ is the same as that of $A_{r, n}$. We need to verify that all entries of $Q_{r, n}$ are non-negative, that all columns sum to 1 , and that all rows sum to 1. It follows immediately from the definition of $Q_{r, n}$ that all entries are non-negative. Because $Q_{r, n}$ is symmetric, it only remains to show that all columns sum to 1 .

First let us consider the case of $0 \leqslant r \leqslant \frac{n-1}{2}$. In this case, for columns $1 \leqslant j \leqslant r$ we have

$$
\sum_{i=1}^{n} q_{i, j}=\sum_{i=1}^{r+1-j} \frac{2}{2 r+1}+\sum_{i=r+2-j}^{r+j} \frac{1}{2 r+1}=1 .
$$

A similar statement holds for columns $n-r+1 \leqslant j \leqslant n$. For the rest of the columns, i.e., for $r+1 \leqslant j \leqslant n-r$, we have

$$
\sum_{i=1}^{n} q_{i, j}=\sum_{i=j-r}^{j+r} \frac{1}{2 r+1}=1 .
$$

Let us now consider the case $\frac{n-1}{2} \leqslant r \leqslant n-1$. For columns $1 \leqslant j \leqslant n-r-1$ we have

$$
\sum_{i=1}^{n} q_{i, j}=\sum_{i=1}^{n-r-j} \frac{2}{n}+\sum_{i=n-r-j+1}^{r+j} \frac{1}{n}=1,
$$

and a symmetric claim holds for columns $r+2 \leqslant j \leqslant n$. Finally, for columns $n-r \leqslant j \leqslant r+1$ we have

$$
\sum_{i=1}^{n} q_{i, j}=\sum_{i=1}^{n} \frac{1}{n}=1 \text {. }
$$

\section{APPENDIX D}

\section{Proof OF THEOREM 13}

Let $r \triangleq \rho \cdot(n-1)$. We distinguish two cases, namely the case $0<\rho \leqslant \frac{1}{2}$ and the case $\frac{1}{2} \leqslant \rho<1$.

Consider the first case, i.e., $0<\rho \leqslant \frac{1}{2}$. We make the following observations about the matrix $Q_{r, n}$ in Definition 11

- $r(r+1)$ entries take on the value $\frac{2}{2 r+1}$,

- $n(2 r+1)-2 r(r+1)$ entries take on the value $\frac{1}{2 r+1}$,

- the remaining entries take on the value 0 .

We obtain

$$
\begin{aligned}
\log _{2} \mid & B_{\rho, n} \mid \\
= & \log _{2} \operatorname{per}\left(A_{\rho, n}\right) \\
\geqslant & \log _{2}\left(\frac{n !}{n^{n}}\right)-r \cdot(r+1) \cdot \frac{2}{2 r+1} \cdot \log _{2}\left(\frac{2}{2 r+1}\right) \\
& \quad-(n(2 r+1)-2 r(r+1)) \cdot \frac{1}{2 r+1} \cdot \log _{2}\left(\frac{1}{2 r+1}\right) \\
= & \log _{2}(n !)-\frac{2 r \cdot(r+1)}{2 r+1}+n \cdot \log _{2}\left(\frac{2 r+1}{n}\right),
\end{aligned}
$$

where the first equality follows from Lemma 2 and where the inequality follows from Theorem 10 with $M \triangleq A_{\rho, n}$ and with $Q \triangleq Q_{r, n}$, where $Q_{r, n}$ was specified in Definition 11. For the asymptotics, we make note of the following:

$$
\begin{aligned}
\log _{2}(n !) & =\log _{2}\left(\left(\frac{n}{e}\right)^{n} \cdot 2^{o(n)}\right) \\
& =n \log _{2}(n)-n \log _{2}(e)+o(n),
\end{aligned}
$$


by Stirling's approximation, as well as

$$
\begin{aligned}
\frac{2 r \cdot(r+1)}{2 r+1} & =\frac{2 \rho(n-1)(\rho(n-1)+1)}{2 \rho(n-1)+1} \\
& =\rho n+o(n)
\end{aligned}
$$

and

$$
\begin{aligned}
n \cdot \log _{2}\left(\frac{2 r+1}{n}\right) & =n \cdot \log _{2}\left(\frac{2 \rho(n-1)+1}{n}\right) \\
& =n \cdot \log _{2}(2 \rho+o(1)) \\
& =n \cdot \log _{2}(2 \rho)+o(n) .
\end{aligned}
$$

Combining these together, asymptotically we get

$$
n \cdot \log _{2}(n)-n \cdot\left[\log _{2}(e)-1+\rho-\log _{2}(\rho)\right]+o(n),
$$

which confirms the expression in the theorem statement.

We now turn to the second case, i.e., $\frac{1}{2} \leqslant \rho<1$. We make the following observations about the matrix $Q_{r, n}$ in Definition 11:

- $(n-r-1)(n-r)$ entries take on the value $\frac{2}{n}$,

- $n^{2}-2(n-r-1)(n-r)$ entries take on the value $\frac{1}{n}$,

- the remaining entries take on the value 0 .

We therefore obtain

$$
\begin{aligned}
\log _{2} \mid & B_{\rho, n} \mid \\
= & \log _{2} \operatorname{per}\left(A_{\rho, n}\right) \\
\geqslant & \log _{2}\left(\frac{n !}{n^{n}}\right)-(n-r-1)(n-r) \cdot \frac{2}{n} \cdot \log _{2}\left(\frac{2}{n}\right) \\
& \quad-\left(n^{2}-2(n-r-1)(n-r)\right) \cdot \frac{1}{n} \cdot \log _{2}\left(\frac{1}{n}\right) \\
= & \log _{2}(n !)-\frac{2 \cdot(n-r-1) \cdot(n-r)}{n},
\end{aligned}
$$

where the first equality follows from Lemma 2 and where the inequality follows from Theorem 10 with $M \triangleq A_{\rho, n}$ and with $Q \triangleq Q_{r, n}$, where $Q_{r, n}$ was specified in Definition 11 For an asymptotic expression we follow the same steps as in the previous case. Thus, asymptotically, the last expression becomes

$$
n \cdot \log _{2}(n)-n \cdot \log _{2}(e)-2 n \cdot(1-\rho)^{2}+o(n),
$$

which confirms the expression in the theorem statement.

\section{APPENDIX E}

\section{Proof of LEMMa 15}

The following lemma collects some results that will prove useful for simplifying some upcoming computations in this appendix and also in Appendix $\mathbf{F}$
Lemma 23. We define

$$
\begin{aligned}
& S_{r}^{(0)} \triangleq \sum_{\ell=0}^{r} \alpha_{r}^{\ell} \quad=\frac{\alpha_{r}^{r+1}-1}{\alpha_{r}-1} \\
& =\frac{\alpha_{r}}{\alpha_{r}-1}, \\
& S_{r}^{(1)} \triangleq \sum_{\ell=0}^{r} \ell \cdot \alpha_{r}^{\ell}=\frac{r \cdot \alpha_{r}^{r+2}-(r+1) \cdot \alpha_{r}^{r+1}+\alpha_{r}}{\left(\alpha_{r}-1\right)^{2}} \\
& =\frac{r \cdot \alpha_{r}^{2}-r-1}{\left(\alpha_{r}-1\right)^{2}} \text {, } \\
& S_{r}^{(2)} \triangleq \sum_{\ell=0}^{r} \ell^{2} \cdot \alpha_{r}^{\ell}=\frac{r^{2} \cdot \alpha_{r}^{r+1}-2 \cdot S_{r}^{(1)}}{\alpha_{r}-1}+\frac{\alpha_{r}^{r+1}-\alpha_{r}}{\left(\alpha_{r}-1\right)^{2}} \\
& =\frac{r^{2} \cdot\left(\alpha_{r}+1\right)}{\alpha_{r}-1}+\frac{1}{\left(\alpha_{r}-1\right)^{2}} \\
& -2 \cdot \frac{r \cdot \alpha_{r}^{2}-r-1}{\left(\alpha_{r}-1\right)^{3}} \text {. }
\end{aligned}
$$

Proof: In each case, the the first summation expression is obtained by standard algebraic techniques, whereas the second summation expression is obtained by simplifying the first summation expression with the help of (7).

We now continue with the proof of Lemma 15 First we note that by definition, $Q_{r, n}$ has the same support as $A_{r, n}$. We need to verify that all entries of $Q_{r, n}$ are non-negative, that all columns sum to 1 , and that all rows sum to 1 . It follows immediately from the definition of $Q_{r, n}$ that all entries are non-negative. Because $Q_{r, n}$ is symmetric, it only remains to show that all columns sum to 1 .

For $1 \leqslant j \leqslant r+1$, we obtain

$$
\begin{aligned}
\sum_{i=1}^{n} q_{i, j} & =C \cdot \sum_{i=1}^{r+1} \alpha_{r}^{(r+1-i)+(r+1-j)}+C \cdot \sum_{i=r+2}^{r+j} \alpha_{r}^{i-j} \\
& =C \cdot \alpha_{r}^{r+1-j} \cdot S_{r}^{(0)}+C \cdot \frac{\alpha_{r}^{r+1}-\alpha_{r}^{r+2-j}}{\alpha_{r}-1} \\
& =C \cdot \alpha_{r}^{r+1-j} \cdot \frac{\alpha_{r}}{\alpha_{r}-1}+C \cdot \frac{\alpha_{r}^{r+1}-\alpha_{r}^{r+2-j}}{\alpha_{r}-1} \\
& =1 .
\end{aligned}
$$

For $n-r \leqslant j \leqslant n$, because of symmetries of the $Q_{r, n}$ matrix, the calculations are analogous to the calculations for $1 \leqslant j \leqslant r+1$.

Finally, for $r+2 \leqslant j \leqslant n-r-1$, we obtain

$$
\sum_{i=1}^{n} q_{i, j}=C \cdot \sum_{i=j-r}^{j+r} \alpha_{r}^{|i-j|}=2 \cdot C \cdot S_{r}^{(0)}-C \cdot \alpha_{r}^{0}=1 .
$$

\section{APPENDIX F}

\section{PROOF OF THEOREM 16}

Recall that $r \triangleq \rho \cdot(n-1)$. In the following, in order to simplify the notation, we define $\alpha \triangleq \alpha_{r}$. As in Appendix D the proof here is based on Lemma 2 and Theorem 10, To this end, we compute the quantity

$$
T \triangleq \sum_{i, j \in[n]} q_{i, j} \log _{2} \frac{q_{i, j}}{m_{i, j}}
$$


where $Q_{r, n}=\left(q_{i, j}\right)$ is the matrix specified in Definition 14 and where $M=\left(m_{i, j}\right)=A_{r, n}$. We decompose $T$ as follows

$$
T=T_{1}+T_{2}+T_{3}+T_{4}+T_{5},
$$

where

$$
\begin{aligned}
& T_{1} \triangleq \sum_{j=1}^{r+1} \sum_{i=1}^{r+1} q_{i, j} \log _{2} \frac{q_{i, j}}{m_{i, j}}, \\
& T_{2} \triangleq \sum_{j=2}^{r+1} \sum_{i=r+2}^{j+r} q_{i, j} \log _{2} \frac{q_{i, j}}{m_{i, j}}, \\
& T_{3} \triangleq \sum_{j=r+2}^{n-r-1} \sum_{i=j-r}^{j+r} q_{i, j} \log _{2} \frac{q_{i, j}}{m_{i, j}}, \\
& T_{4} \triangleq \sum_{j=n-r}^{n-1} \sum_{i=j-r}^{n-r-1} q_{i, j} \log _{2} \frac{q_{i, j}}{m_{i, j}}, \\
& T_{5} \triangleq \sum_{j=n-r}^{n} \sum_{i=n-r}^{n} q_{i, j} \log _{2} \frac{q_{i, j}}{m_{i, j}} .
\end{aligned}
$$

Because of the symmetries of the setup, we have $T_{1}=T_{5}$ and $T_{2}=T_{4}$. Therefore, we only need to determine $T_{1}, T_{2}$, and $T_{3}$. We get, using the notation from Lemma 23 in Appendix E

$$
\begin{aligned}
T_{1}= & \sum_{j=1}^{r+1} \sum_{i=1}^{r+1} C \cdot \alpha^{(r+1-i)+(r+1-j)} \log _{2}\left(C \cdot \alpha^{(r+1-i)+(r+1-j)}\right) \\
= & C \cdot\left(S_{r}^{(0)}\right)^{2} \cdot \log _{2}(C)+2 \cdot C \cdot S_{r}^{(0)} \cdot S_{r}^{(1)} \cdot \log _{2}(\alpha), \\
T_{2}= & \sum_{j=2}^{r+1} \sum_{i=r+2}^{j+r} q_{i, j} \log _{2} \frac{q_{i, j}}{m_{i, j}} \\
= & \sum_{\ell=0}^{r} \ell \cdot C \cdot \alpha^{\ell} \cdot \log _{2}\left(C \cdot \alpha^{\ell}\right) \\
= & C \cdot S_{r}^{(1)} \cdot \log (C)+C \cdot S_{r}^{(2)} \cdot \log (\alpha), \\
T_{3}= & \sum_{j=r+2}^{n-r-1} \sum_{i=j-r}^{j+r} q_{i, j} \log _{2} \frac{q_{i, j}}{m_{i, j}} \\
= & ((n-r-1)-(r+2)+1) \cdot \sum_{\ell=-r}^{r} C \cdot \alpha^{|\ell|} \cdot \log _{2}\left(C \cdot \alpha^{|\ell|}\right) \\
= & (n-2 r-2) \cdot C \cdot\left(2 S_{r}^{(0)}-1\right) \cdot \log (C) \\
& +(n-2 r-2) \cdot C \cdot\left(2 S_{r}^{(1)}-0\right) \cdot \log (\alpha) .
\end{aligned}
$$

Recall that $\alpha$ satisfies $\alpha^{r+1}-\alpha-1=0$. The following lemma gives an approximation of $\alpha$ which is precise enough for the upcoming computations.

\section{Lemma 24. It holds that}

$$
\alpha=1+\frac{\ln (2)}{r}+o(1 / r) .
$$

Proof: Consider the following two functions

$$
\begin{aligned}
& \underline{g}(r) \triangleq \underline{\alpha}^{r+1}-\underline{\alpha}-\left.1\right|_{\underline{\alpha}=1+\frac{\ln (2)}{r+1}}, \\
& \bar{g}(r) \triangleq \bar{\alpha}^{r+1}-\bar{\alpha}-\left.1\right|_{\bar{\alpha}=1+\frac{\ln (2)}{r}} .
\end{aligned}
$$

One can show that $g(r)$ is a strictly increasing function of $r>0$, ultimately converging to 0 as $r \rightarrow \infty$, and that $\bar{g}(r)$ is a strictly decreasing function of $r>0$, ultimately converging to 0 as $r \rightarrow \infty$. (We omit the straightforward, but tedious, details.)

From these observations, it follows that $\alpha$ satisfies

$$
1+\frac{\ln (2)}{r+1}=\underline{\alpha} \leqslant \alpha \leqslant \bar{\alpha}=1+\frac{\ln (2)}{r},
$$

which implies the expression in the lemma statement.

In the following, we will therefore use $\alpha=1+\frac{\ln (2)}{r}+$ $o(1 / r)$. With this, we obtain $C=\frac{\ln (2)}{2 \rho n}+o(1 / n)$.

Putting everything together, and using Lemma 2 and Theorem 10, we get

$$
\begin{aligned}
& \log _{2}\left|B_{\rho, n}\right| \\
\geqslant & \log _{2}\left(\frac{n !}{n^{n}}\right)-T \\
= & n \cdot \log _{2}(n) \\
& -n \cdot\left[\left(\log _{2}(e)-1\right) \cdot 2 \rho-\log _{2}(\rho)-\log _{2}\left(\log _{2}(e)\right)+1\right] \\
& +o(n) .
\end{aligned}
$$

\section{APPENDIX G \\ Proof OF LEMMA 18}

We begin by noting that the support of $Q_{r, n}$ is the same as that of $A_{r, n}$. We need to verify that all entries of $Q_{r, n}$ are non-negative, that all columns sum to 1 , and that all rows sum to 1. It follows immediately from the definition of $Q_{r, n}$ that all entries are non-negative. Because $Q_{r, n}$ is symmetric, it only remains to show that all columns sum to 1 . In order to simplify the notation, in the following we set $\alpha \triangleq \alpha_{r, n}$.

For $1 \leqslant j \leqslant n-r$, we obtain

$$
\begin{aligned}
\sum_{i=1}^{n} q_{i, j}=C \cdot \alpha^{n-r-j} \cdot\left(\alpha^{n-r-1}+\cdots+\alpha^{2}+\alpha+1\right. \\
+(2 r-n) \cdot 1 \\
\left.\quad+1+\alpha+\alpha^{2}+\cdots+\alpha^{j-1}\right) \\
=C \cdot \alpha^{n-r-j} \cdot\left(\frac{\alpha^{n-r}-1}{\alpha-1}+2 r-n+\frac{\alpha^{j}-1}{\alpha-1}\right) \\
=\alpha^{-j} \cdot\left(\alpha^{n-r}-1+(2 r-n) \cdot(\alpha-1)+\alpha^{j}-1\right) \\
=1,
\end{aligned}
$$

where the third equality follows from plugging in the expression for $C$ from 9 and where the fourth equality follow from using (11) to simplify the expression.

For $n-r+1 \leqslant j \leqslant r$, we obtain

$$
\sum_{i=1}^{n} q_{i, j}=\sum_{i=1}^{n} q_{i, n-r}=1,
$$

where the first equality follows from $q_{i, j}=q_{i, n-r}, i \in[n]$, and where the second equality follows from the above computations.

For $r+1 \leqslant j \leqslant n$, we can use the symmetries of the matrix $Q_{r, n}$ and the above computations to argue that $\sum_{i=1}^{n} q_{i, j}=1$. 


\section{APPENDIX H}

Proof OF LEMMA 19

In the following, in order to simplify the notation, we define $\alpha \triangleq \alpha_{r, n}$. As in Appendices $\mathrm{D}$ and $\mathrm{F}$ the proof here is based on Lemma 2 and Theorem 10 To this end, we compute the quantity

$$
T \triangleq \sum_{i, j \in[n]} q_{i, j} \log _{2} \frac{q_{i, j}}{m_{i, j}},
$$

where $Q_{r, n}$ is the matrix specified in Definition 17 and where $M=A_{r, n}$. We get

$$
\begin{aligned}
T= & \sum_{i, j \in[n]} q_{i, j} \cdot \log _{2}\left(C \cdot \exp _{2}\left(\lambda_{i}\right) \cdot \exp _{2}\left(\lambda_{j}^{\prime}\right)\right) \\
= & n \cdot \log _{2}(C)+\sum_{i \in[n]} \lambda_{i}+\sum_{j \in[n]} \lambda_{j}^{\prime} \\
= & n \cdot \log _{2}(\alpha-1)-n \cdot(n-r) \cdot \log _{2}(\alpha) \\
& +2 \cdot(n-r) \cdot(n-r-1) \cdot \log _{2}(\alpha) \\
= & n \cdot \log _{2}(\alpha-1)-(n-r) \cdot(2 r-n+2) \cdot \log _{2}(\alpha) .
\end{aligned}
$$

Using Lemma 2 and Theorem 10 we therefore obtain

$$
\begin{aligned}
\log _{2} \mid & \left|\beta_{\rho, n}\right| \\
= & \log _{2} \operatorname{per}\left(A_{\rho, n}\right) \\
\geqslant & \log _{2}\left(\frac{n !}{n^{n}}\right)-T \\
= & \log _{2}(n !)-n \log _{2}(n)-n \cdot \log _{2}(\alpha-1) \\
& \quad+(n-r) \cdot(2 r-n+2) \cdot \log _{2}(\alpha) .
\end{aligned}
$$

\section{APPENDIX I}

Proof of LEMMA 20

Let $\alpha \triangleq \alpha_{r, n}$. We define the function $f: \mathbb{R} \rightarrow \mathbb{R}$,

$$
f(x)=x^{n-r}+(2 r-n) \cdot x-(2 r-n+2) .
$$

By definition, $\alpha$ is the unique positive root of $f(x)$. We note that

$$
\begin{aligned}
f(1) & =1+(2 r-n)-(2 r-n+2)=-1<0, \\
f\left(2^{\frac{1}{n-r}}\right) & =2+(2 r-n) \cdot 2^{\frac{1}{n-r}}-(2 r-n+2)>0 .
\end{aligned}
$$

It follows that

$$
\alpha \in\left[1,2^{\frac{1}{n-r}}\right] .
$$

We rewrite $\alpha$ by introducing a real parameter $t \in[0,1]$,

$$
\alpha=1+t \cdot\left(2^{\frac{1}{n-r}}-1\right) .
$$

In order to find the value of $t$ we need to solve

$$
\begin{aligned}
0 & =f(\alpha) \\
& =\left(1+t \cdot\left(2^{\frac{1}{n-r}}-1\right)\right)^{n-r}+(2 r-n) \cdot t \cdot\left(2^{\frac{1}{n-r}}-1\right)-2 .
\end{aligned}
$$

Solving 15 is not easy. Instead of taking the direct route, we observe that

$$
\lim _{n \rightarrow \infty} f(\alpha)=2^{t}+t \cdot \frac{(2 \rho-1) \ln (2)}{1-\rho}-2 .
$$

We conveniently define the right-hand side of (15) as $g(t)$, and the right-hand side of (16) as $\hat{g}(t)$. We would like to find $t^{*}$ such that $g\left(t^{*}\right)=0$, which appears to be a difficult task. Instead, we find $\hat{t}$ such that $\hat{g}(\hat{t})=0$, and claim that it is not too far from $t^{*}$.

We first note that $\hat{t}$ from (12) indeed satisfies $\hat{g}(\hat{t})=0$. This is done by expanding $\hat{g}(\hat{t})$ and remembering that $e^{W(z)}=$ $\frac{z}{W(z)}$.

We now need to bound $\left|t^{*}-\hat{t}\right|$. Since $\hat{g}(t)$ is continuous and monotone increasing in $[0,1]$,

$$
\left|t^{*}-\hat{t}\right| \leqslant \frac{\max _{x \in[0,1]}|g(x)-\hat{g}(x)|}{\min _{x \in[0,1]}\left|\frac{d}{d x} \hat{g}(x)\right|} .
$$

It is easy to verify that

$$
|g(x)-\hat{g}(x)|=\Theta\left(n^{-1}\right), \quad x \in[0,1],
$$

by noting that

$$
\begin{aligned}
& \lim _{n \rightarrow \infty} n \cdot(g(x)-\hat{g}(x)) \\
& =\frac{x \ln (2)}{2(1-\rho)^{2}} \\
& \quad \cdot\left((2 \rho-1) \cdot \ln (2)-2^{x} \cdot(x-1) \cdot(1-\rho) \cdot \ln (2)-2 \rho\right) .
\end{aligned}
$$

Furthermore, we get

$$
\min _{x \in[0,1]}\left|\frac{d}{d x} \hat{g}(x)\right|=\frac{\rho \cdot \ln (2)}{1-\rho} .
$$

Combining everything together, we get that the sought after $t^{*}$, for which $g\left(t^{*}\right)=0$, is

$$
t^{*}=\hat{t}+\Theta\left(n^{-1}\right)
$$

which completes the proof the lemma.

\section{APPENDIX J}

ProOF OF THEOREM 21

Let $\alpha \triangleq \alpha_{r, n}$. With the help of Theorem 10 we obtain

$$
\begin{aligned}
\log _{2}\left|B_{r, n}\right| \geqslant & \log _{2}(n !)-n \cdot \log _{2}(n)-n \cdot \log _{2}(\alpha-1) \\
& +(n-r) \cdot(2 r-n+2) \cdot \log _{2}(\alpha) \\
= & -n \cdot \log _{2}(e)-n \cdot \log _{2}(\alpha-1) \\
& +(n-r) \cdot(2 r-n+2) \cdot \log _{2}(\alpha)+o(n),
\end{aligned}
$$

where the last equality is due to Stirling's approximation. In order to evaluate $-n \cdot \log _{2}(\alpha-1)$, we use Lemma 20 and get

$$
\begin{aligned}
-n \cdot \log _{2}(\alpha-1)= & -n \cdot \log _{2}\left[\left(\hat{t}+\Theta\left(n^{-1}\right)\right) \cdot\left(2^{\frac{1}{n-r}}-1\right)\right] \\
= & -n \cdot \log _{2}\left(\hat{t}+\Theta\left(n^{-1}\right)\right) \\
& -n \cdot \log _{2}\left(\frac{1}{(1-\rho) n \log _{2}(e)}+O\left(n^{-2}\right)\right) \\
= & -n \cdot \log _{2}(\hat{t})+n \cdot \log _{2}\left(\log _{2}(e)\right) \\
& +n \cdot \log _{2}(1-\rho)+n \cdot \log _{2}(n)+o(n),
\end{aligned}
$$

where the derivation uses a Taylor series expansion of $2^{x}$ around $x=0$. 
Similarly, $(n-r) \cdot(2 r-n+2) \cdot \log _{2}(\alpha)$ can be rewritten as

$$
\begin{aligned}
(n-r) & \cdot(2 r-n+2) \cdot \log _{2}(\alpha) \\
= & (n-r) \cdot(2 r-n+2) \\
& \cdot \log _{2}\left[1+\left(\hat{t}+\Theta\left(n^{-1}\right)\right) \cdot\left(2^{\frac{1}{n-r}}-1\right)\right] \\
= & (n-r) \cdot(2 r-n+2) \\
& \cdot \log _{2}\left[1+\left(\hat{t}+\Theta\left(n^{-1}\right)\right)\right. \\
& \left.\cdot\left(\frac{1}{(1-\rho) n \log _{2}(e)}+O\left(n^{-2}\right)\right)\right] \\
= & n^{2} \cdot(1-\rho) \cdot(2 \rho-1) \cdot \hat{t} \cdot \frac{1}{(1-\rho) \cdot n}+o(n) \\
= & n \cdot(2 \rho-1) \cdot \hat{t}+o(n),
\end{aligned}
$$

where the derivation uses a Taylor series expansion of $2^{x}$ around $x=0$ and a Taylor series expansion of $\log _{2}(1+y)$ around $y=0$.

Combining everything together we get

$$
\log _{2}\left|B_{r, n}\right| \geqslant \log _{2}\left(\varphi_{3}(\rho, n)\right)+o(n),
$$

as claimed.

\section{REFERENCES}

[1] R. F. Bailey, "Error-correcting codes from permutation groups," Discrete Math., vol. 309, no. 13, pp. 4253-4265, 2009.

[2] A. Barg and A. Mazumdar, "Codes in permutations and error correction for rank modulation," IEEE Trans. Inform. Theory, vol. 56, no. 7, pp. 3158-3165, Jul. 2010.

[3] I. F. Blake, "Permutation codes for discrete channels," IEEE Trans. Inform. Theory, vol. 20, pp. 138-140, 1974.

[4] I. F. Blake, G. Cohen, and M. Deza, "Coding with permutations," Inform. and Control, vol. 43, pp. 1-19, 1979.

[5] S. Buzaglo and T. Etzion, "Perfect permutation codes with the Kendall's $\tau$-metric," in Proc. IEEE Int. Symp. Inf. Theory, Honolulu, HI, USA, Jun. 2014, pp. 2391-2395

[6] S. Buzaglo, E. Yaakobi, and T. Etzion, "Systematic codes for rank modulation," in Proc. IEEE Int. Symp. Inf. Theory, Honolulu, HI, USA, Jun. 2014, pp. 2386-2390.

[7] P. J. Cameron, "Permutation codes," European J. of Combin., vol. 31, pp. 482-490, 2010.

[8] P. J. Cameron and I. M. Wanless, "Covering radius for sets of permutations," Discrete Math., vol. 293, pp. 91-109, 2005.

[9] H. D. Chadwick and L. Kurz, "Rank permutation group codes based on Kendall's correlation statistic," IEEE Trans. Inform. Theory, vol. IT-15, no. 2, pp. 306-315, Mar. 1969.

[10] G. Cohen, I. Honkala, S. Litsyn, and A. Lobstein, Covering Codes. North-Holland, 1997.

[11] J. H. Conway and N. J. A. Sloane, Sphere Packings, Lattices, and Groups. Springer Verlag, 1988.

[12] M. Deza and H. Huang, "Metrics on permutations, a survey," J. Comb. Inf. Sys. Sci., vol. 23, pp. 173-185, 1998.

[13] M. Deza and S. A. Vanstone, "Bounds for permutation arrays," J. of Statistical Planning and Inference, vol. 2, no. 2, pp. 197-209, 1978.

[14] M. Deza and P. Frankl, "On maximal numbers of permutations with given maximal or minimal distance," J. Combin. Theory Ser. A, vol. 22, 1977.

[15] G. P. Egorychev, "Proof of the van der Waerden conjecture for permanents (in Russian)," Sibirsk. Mat. Zh., vol. 22, no. 6, pp. 65-71, Nov.-Dec. 1981.

[16] D. I. Falikman, "Proof of the Van der Waerden conjecture regarding the permanent of a doubly stochastic matrix," Mathematical Notes, vol. 29, no. 6, pp. 475-479, 1981.

[17] F. Farnoud, M. Schwartz, and J. Bruck, "Bounds for permutation ratedistortion," IEEE Trans. Inform. Theory, vol. 62, no. 2, pp. 703-712, Feb. 2016.
[18] F. Farnoud, V. Skachek, and O. Milenkovic, "Error-correction in flash memories via codes in the Ulam metric," IEEE Trans. Inform. Theory, vol. 59, no. 5, pp. 3003-3020, May 2013.

[19] F. Göloğlu, J. Lember, A.-E. Riet, and V. Skachek, "New bounds for permutation codes in Ulam metric," in Proc. 2015 IEEE Int. Symp. Inf. Theory, Hong Kong, China SAR, Jun. 2015, pp. 1726-1730.

[20] R. L. Graham, D. E. Knuth, and O. Patashnik, Concrete Mathematics: A Foundation for Computer Science. Addison-Wesley, 1994.

[21] V. J. W. Guo and Y. Yang, "Proof of a conjecture of Kløve on permutation codes under the Chebychev distance," Designs, Codes and Cryptography, 2016.

[22] L. Gurvits, "Unleashing the power of Schrijver's permanental inequality with the help of the Bethe approximation," CoRR, available online under http://arxiv.org/abs/1106.2284, Jun. 2011.

[23] B. Huang and T. Jebara, "Approximating the permanent with belief propagation," CoRR, available online under http://arxiv.org/abs / 0908.1769, Aug. 2009.

[24] A. Jiang, R. Mateescu, M. Schwartz, and J. Bruck, "Rank modulation for flash memories," IEEE Trans. Inform. Theory, vol. 55, no. 6, pp. 2659-2673, Jun. 2009.

[25] A. Jiang, M. Schwartz, and J. Bruck, "Correcting charge-constrained errors in the rank-modulation scheme," IEEE Trans. Inform. Theory, vol. 56, no. 5, pp. 2112-2120, May 2010.

[26] P. Keevash and C. Y. Ku, "A random construction for permutation codes and the covering radius," Designs, Codes and Cryptography, vol. 41, pp. 79-86, 2006.

[27] T. Kløve, "Spheres of permutations under the infinity norm - permutations with limited displacement," University of Bergen, Bergen, Norway, Tech. Rep. 376, Nov. 2008.

[28] — "Generating functions for the number of permutations with limited displacement," Elec. J. of Comb., vol. 16, pp. 1-11, 2009.

[29] _ _Lower bounds on the size of spheres of permutations under the Chebychev distance," Designs, Codes and Cryptography, vol. 59, no. 1-3, pp. 183-191, 2011.

[30] T. Kløve, T.-T. Lin, S.-C. Tsai, and W.-G. Tzeng, "Permutation arrays under the Chebyshev distance," IEEE Trans. Inform. Theory, vol. 56, no. 6, pp. 2611-2617, Jun. 2010.

[31] D. H. Lehmer, "Permutations with strongly restricted displacements," in Combinatorial Theory and its Applications II, P. Erdős, A. Rényi, and V. T. Sós, Eds. Amsterdam, The Netherlands: North Holland, 1970, pp. 273-291.

[32] N. Linial, A. Samorodnitsky, and A. Wigderson, "A deterministic strongly polynomial algorithm for matrix scaling and approximate permanents," Combinatorica, vol. 20, no. 4, pp. 545-568, 2000.

[33] F. J. MacWilliams and N. J. A. Sloane, The Theory of Error-Correcting Codes. North-Holland, 1978.

[34] A. Mazumdar, A. Barg, and G. Zémor, "Constructions of rank modulation codes," IEEE Trans. Inform. Theory, vol. 59, no. 2, pp. 1018-1029, Feb. 2013.

[35] J. Quistorff, "A survey on packing and covering problems in the Hamming permutation space," Elec. J. of Comb., vol. 13, pp. 1-13, 2006.

[36] M. Schwartz, "Efficiently computing the permanent and Hafnian of some banded Toeplitz matrices," Linear Algebra and its Applications, vol. 430, no. 4, pp. 1364-1374, Feb. 2009.

[37] M. Schwartz and I. Tamo, "Optimal permutation anticodes with the infinity norm via permanents of $(0,1)$-matrices," J. Combin. Theory Ser. A, vol. 118, pp. 1761-1774, 2011.

[38] M. Schwartz and P. O. Vontobel, "Bounds on the size of balls over permutations with the infinity metric," in Proc. 2015 IEEE Int. Symp. Inf. Theory, Hong Kong, China SAR, Jun. 2015, pp. 1731-1735.

[39] M.-Z. Shieh and S.-C. Tsai, "Decoding frequency permutation arrays under Chebyshev distance," IEEE Trans. Inform. Theory, vol. 56, no. 11, pp. 5730-5737, Nov. 2010.

[40] R. Sinkhorn, "A relationship between arbitrary positive matrices and doubly stochastic matrices," Annals of Mathematical Statistics, vol. 35 , no. 2, pp. 876-879, Jun. 1964.

[41] R. P. Stanley, Enumerative Combinatorics: Volume 1 (2nd Edition). Cambridge University Press, 2011.

[42] I. Tamo and M. Schwartz, "Correcting limited-magnitude errors in the rank-modulation scheme," IEEE Trans. Inform. Theory, vol. 56, no. 6 , pp. 2551-2560, Jun. 2010.

[43] - "On the labeling problem of permutation group codes for the infinity metric," IEEE Trans. Inform. Theory, vol. 58, no. 10, pp. 65956604, Oct. 2012.

[44] P. O. Vontobel, "The Bethe permanent of a non-negative matrix," IEEE Trans. Inform. Theory, vol. 59, no. 3, pp. 1866-1901, Mar. 2013. 
[45] — "The Bethe and Sinkhorn approximations of the pattern maximum likelihood estimate and their connections to the Valiant-Valiant estimate," in Proc. 2014 Inf. Theory and Appl. Workshop (ITA2014), San Diego, CA, USA, Feb. 2014.

[46] D. Wang, A. Mazumdar, and G. W. Wornell, "Compression in the space of permutations," IEEE Trans. Inform. Theory, vol. 61, no. 12, pp. 64176431, Dec. 2015.

[47] Y. Yehezkeally and M. Schwartz, "Snake-in-the-box codes for rank modulation," IEEE Trans. Inform. Theory, vol. 58, no. 8, pp. 54715483, Aug. 2012.

[48] — "Limited-magnitude error-correcting Gray codes for rank modulation," in Proc. 2016 IEEE Int. Symp. Inf. Theory, Barcelona, Spain, Jun. 2016, pp. 2829-2833.

[49] H. Zhou, M. Schwartz, A. Jiang, and J. Bruck, "Systematic errorcorrecting codes for rank modulation," IEEE Trans. Inform. Theory, vol. 61, no. 1, pp. 17-32, Jan. 2015. 